\title{
Erratum
}

\section{Surface induced mixed state of a superconducting film}

\section{B.J. Yuan}

Clarenville Campus, Eastern College of Applied Arts, Technology and continuing Education, Clarenville, Newfoundland, Canada 10E1Jo

This note is to correct some mistakes in a report published in this journal in 1994, which were due to an error in my computer programme. It affects the results of four figures in Sect. III, namely, Figs. 5a, b, 6 and 7. This error affects
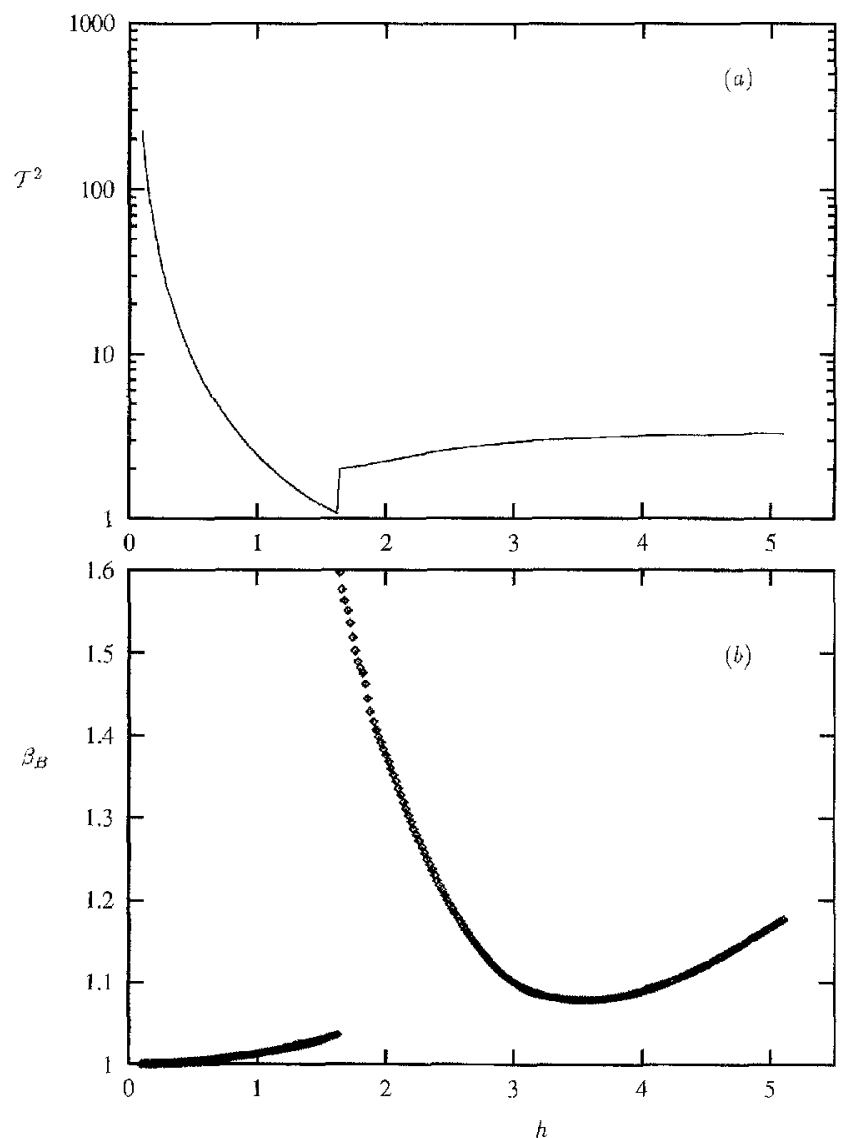

Fig. 5. a shows the ratio $T^{2}=\left(F^{4}\right) /\left(F\left|\mathbf{b}_{z}^{s}\right| F\right) 1 / \lambda^{*}$ as a function of $h$, which enhances the $\kappa_{2}$ value due to the surface effect. b shows variations of $\beta_{B}$ as a function of $h$ for film geometry only the detailed description of some numerical results from previous calculations (see the erratum table below); it has absolutely no significance for the theoretical analysis Z. Phys. B 96, 165 (1994)

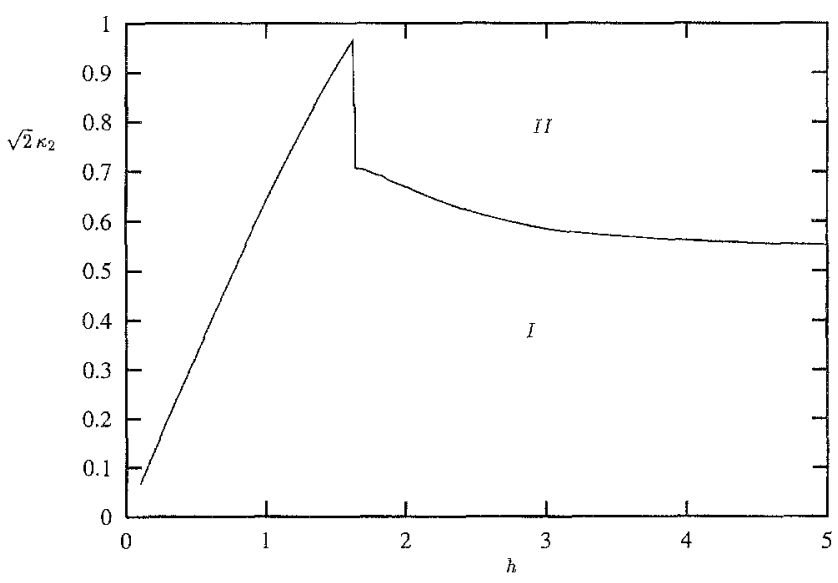

Fig. 6. Phase diagram in parallel geometry. Domain I: The transition at $H_{u}$ is first order. Domain II: The transition at $H_{u}$ is second order

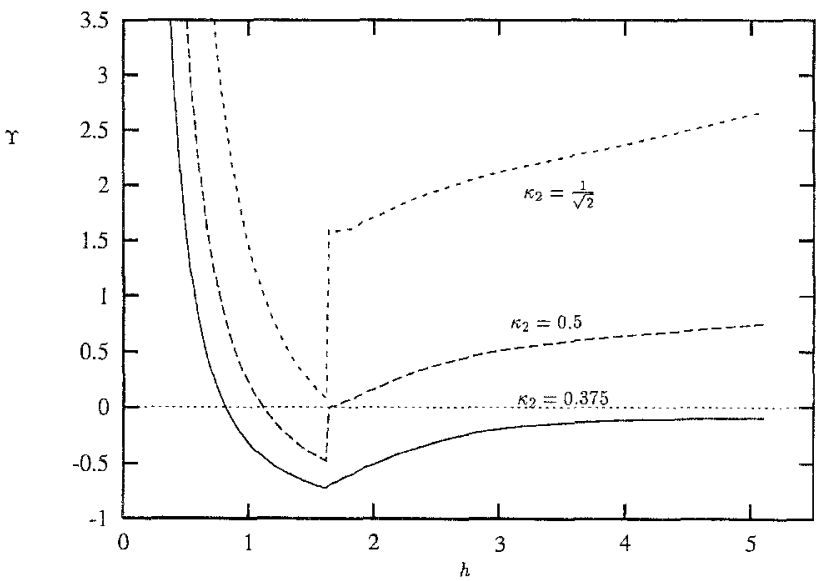

Fig. 7. Plot shows $\gamma$ in a film for three values of $\kappa_{2}$ 


\begin{tabular}{|c|c|c|c|c|c|}
\hline Section & Page & Column & Paragraph & Line & Correction \\
\hline III & 171 & $\mathrm{r}$ & 2nd & 15 & 1.07 to $2.90 \rightarrow 1.07$ to 2.00 \\
\hline III & 172 & $\mathrm{r}$ & 5 th & 5 & $\sqrt{2} \kappa_{2}<0.53 \rightarrow \sqrt{2} \kappa_{2}<0.55$ \\
\hline III & 172 & $\mathrm{r}$ & 5 th & 10 & $0.59<\sqrt{2} \kappa_{2}<1 \rightarrow 0.71<\sqrt{2} \kappa_{2}<1$ \\
\hline III & 172 & r & 5 th & $25-30$ & $\begin{aligned} 0.53 & <\sqrt{2} \kappa_{2}<0.56 \ldots \text { specified on } \sqrt{2} \kappa_{2} . \\
& \rightarrow 0.55<\sqrt{2} \kappa_{2}<0.71 \text {, a II-I-II film. }\end{aligned}$ \\
\hline III & 173 & 1 & 6 th & 4 & $\beta_{B} \approx 1.1 \rightarrow \beta_{B} \approx 1.6$ \\
\hline III & 173 & 1 & 6th & 11 & $h \approx 2.36 \rightarrow h \approx 3.5$ \\
\hline III & 174 & 1 & 10 th & $10-22$ & $\begin{array}{l}\text { It is seen... Type II superconductor } \\
\text { Void }\end{array}$ \\
\hline
\end{tabular}

Zeitschrift für Physik B is delivered to some institutions in the former Soviet Union through a program initiated and administered by the European Physical Society and supported by the publisher. This program is funded by INTAS, the international association for the promotion of cooperation with scientists from the independent States of the former Soviet Union. Members of INTAS are the European Union, Austria, Belgium, Denmark, Finland, France, Germany, Greece, Ireland, Italy, Luxembourg, The Netherlands, Portugal, Spain, United Kingdom and Switzerland. (INTAS, rue du Luxembourg 14A, 1040 Bruxelles, Belgique). 